\title{
POSSIBILITY OF ACETYLCHOLINESTERASE OVEREXPRESSION IN ALZHEIMER DISEASE PATIENTS AFTER THERAPY WITH ACETYLCHOLINESTERASE INHIBITORS
}

\begin{abstract}
Aľ̌běta Kračmarová ${ }^{1}$, Lucie Drtinová ${ }^{1}$, Miroslav Pohanka ${ }^{2}$
Department of Toxicology and Military Pharmacy ${ }^{1}$, Department of Molecular Pathology and Biology ${ }^{2}$, Faculty of Military Health Sciences, University of Defense, Czech Republic

Summary: Acetylcholinesterase is an enzyme responsible for termination of excitatory transmission at cholinergic synapses by the hydrolyzing of a neurotransmitter acetylcholine. Nowadays, other functions of acetylcholinesterase in the organism are considered, for example its role in regulation of apoptosis. Cholinergic nervous system as well as acetylcholinesterase activity is closely related to pathogenesis of Alzheimer disease. The mostly used therapy of Alzheimer disease is based on enhancing cholinergic function using inhibitors of acetylcholinesterase like rivastigmine, donepezil or galantamine. These drugs can influence not only the acetylcholinesterase activity but also other processes in treated organism. The paper is aimed mainly on possibility of increased expression and protein level of acetylcholinesterase caused by the therapy with acetylcholinesterase inhibitors.
\end{abstract}

Keywords: Acetylcholinesterase; Neurodegeneration; Alzheimer disease; Acetylcholine; Inhibition

\section{Introduction}

Acetylcholinesterase (AChE; E.C. 3.1.1.7) is an ubiquitous from class of serine hydrolases. In the body, it is responsible for hydrolyzing neurotransmitter acetylcholine. The second important cholinesterase - butyrylcholinesterase (BChE; E.C. 3.1.1.8.) - is known in organism as well. $\mathrm{AChE}$ and $\mathrm{BChE}$ are homologous enzymes. Genes encoding $\mathrm{AChE}$ and $\mathrm{BChE}$ are located on chromosome $7 \mathrm{q} 22$ and on chromosome 3q26 $(1,2)$.

Alzheimer's disease (AD) is the most common form of dementia which is manifested by loss of short-term memory, spatial disorientation, progressive loss of cognitive function, decrease of intellect and some other minor manifestations (3). It is estimated that about 36 million people worldwide suffer from dementia (mostly AD) and this number continues to grow (4). $\mathrm{AD}$ is unpredictable, multifactorial disease of unknown origin and casual therapy. In most post-mortem investigated brains, loss of nerve tissue, oxidative damage induced by inflammation and neuronal decay, extracellular aggregated amyloid beta in amyloid plaque and hyperphosphorylated tau protein tangles are typical findings of $\mathrm{AD}$ (5). The disease always ends lethally after $2-10$ years from diagnosis. Currently, the only available drugs for $\mathrm{AD}$ cause symptomatic treatment and most treated patients build a tolerance to them over time. The effect of drugs used in the treatment of $\mathrm{AD}$ is based on inhibition of $\mathrm{AChE}$ (donepezil, galantamine, rivastigmine) or on antagonism on N-methyl-D-aspartate (NMDA) receptor (memantine) (4).

\section{Structure of AChE}

AChE belongs to the $\alpha / \beta$-fold family of proteins, since it consists of stranded central $\beta$-sheets surrounded by $\alpha$-helices $(6,7)$.

The crystal structure of Torpedo californica AChE (TcAChE) was visualized at the first. Discovery of the human AChE ( $h A C h E)$ structure followed and similarities between $h \mathrm{AChE}$ and $T c \mathrm{AChE}$ were revealed (6). Catalytic active site (CAS), active site gorge and peripheral anionic site (PAS) compose three main parts of the catalytic subunit. CAS consists of two major subunits, the "esteratic" and the "catalytic anionic" subsites. The crystal structure of $T c \mathrm{AChE}$ showed that the "esteratic" subsite involves Ser200, Glu327 and His440 residues, so called catalytic triad (6). All the three triad residues occur within highly conserved regions of the sequence and are typical for the both cholinesterases $(6,7)$. Within the esteratic subsite, an acyl pocket and an oxyanion hole are situated. The acyl pocket is a spot where the acyl group of choline esters is held in place during catalysis. According to the enzyme model, the oxyanion hole could be formed by the main chain amide nitrogens of glycine and alanine, interacting in such way with carbonyl oxygen in the acetylcholine. The anionic subsite of CAS interacts with the charged quaternary group of the choline moiety, and is believed to be the binding site for both quaternary ligands, such as edrophonium and $\mathrm{N}$-methylacridinium, which act as competitive inhibitors, and for quaternary oximes, which serve as effective reactivators of organophosphate-inhibited 
ChE. (6) Quaternary nitrogens probably interact preferentially with the $\pi$ electrons of aromatic rings. Moreover the aromatic groups interact more strongly with quaternary ammonium ligands than with isosteric uncharged ligands, presumably due to the polarizability of the ion $(6,8)$.

The characteristic feature of the AChE structure is a deep and narrow gorge, about $20 \AA$ long ending in the middle of the enzyme molecule. It is called "active-site gorge" because it contains the catalytic triad on the bottom. This gorge is composed of the 14 aromatic residues rings which can be differing in compliance with origin of the AChE. The presence of aromatic hydrophobic residues may help to introduce acetylcholine to the CAS, thus accounting for the rapid turnover rate of the enzyme. In addition to the two subsites of the catalytic centre, AChE was shown to have other additional binding site: "peripheral anionic site" (PAS) serving as a selective gate for acetylcholine but other ligands having aromaticity or quaternary nitrogens can also bind. Residues in positions 251-264 and 270-278 were identified as a part of the PAS. Substrate binding to PAS is the initial step in the catalytic pathway (9).

The alternative splicing, transcriptional, post-transcriptional and post-translational modifications give increase to the unusually rich molecular polymorphism of AChE. This means, that the human AChE exists in multiple molecular forms. It appears in globular forms G1, G2 (131 kDa dimertwo monomers linked by a disulfide bond Cys-Cys) and G4 (275 kDa tetramer - two G2 connected by the hydrophobic interactions), which consist of one, two or four subunits of AChE. Other types of shape of an extended molecule are termed asymmetric forms of AChE (10). Asymmetric AChE forms consist of one or three subunits of AChE. A12 is the most frequent form $(811 \mathrm{kDa})$ consisting of 12 subunits. In the mammalian brain, AChE is most frequently found in the globular tetrameric form $\mathrm{G} 4$ which is anchored by glycolipid bridge $(20 \mathrm{kDa})$ to cell membrane (11). G4 form is accompanied by a small amount of monomeric G1 form freely situated in the vicinity of the membrane (12). Asymmetric collagen-tailed $\mathrm{AChE}$ form is found only in peripheral nerves and muscles of vertebrates, while proline-rich membrane anchored G4 AChE is found mostly in mammalian brains (13). The different isoforms of AChE could have significantly different catalytic properties (2).

\section{Function of AChE in organism}

The principal biological role of AChE is termination of excitatory transmission at cholinergic synapses by the rapid hydrolysis of the neurotransmitter acetylcholine. AChE has a huge dipole moment which can attract the positively charged acetylcholine. The acetylcholine is trapped at the mouth of the gorge and it diffuses rapidly down to the active site (6). The catalytic reaction by AChE proceeds with the nucleophilic attack on the carbonyl carbon, acylating the enzyme and liberating choline. This is followed by a rapid hydrolysis of the acylated enzyme yielding to the release corresponding acid and to the restoration of the esteratic subsite of active site (9). The turnover number of AChE is one of the smallest and $\mathrm{AChE}$ belongs between the fastest enzymes (2).

Expression and activity of acetylcholine synthesizing enzyme choline acetyltransferase (ChAT) and acetylcholine degrading enzymes $\mathrm{AChE}$ and $\mathrm{BChE}$ is known from various non-nervous tissues. The fact suggests the involvement of a cholinergic system in regulation of functions other than synaptic transmission (14).

For example Zhang et al. found increased level of AChE in various cell types (human fibroblasts, leukemia, cervical carcinoma or neuroblastoma cells, murine leukemia and endothelial cells, rat liver and smooth muscle cells and megakaryocytes, swine and bovine primary endothelial cells) during an apoptotic process. They inferred that $\mathrm{AChE}$ role in these cells has been to regulate apoptosis (15). AChE expression was also observed in neuronal and non-neuronal cells by Thullbery et al. Both neuronal and non-neuronal cells expressed equivalent levels of AChE protein however the catalytic activity of AChE was significantly higher in neuronal cells. In non-neuronal cells, the localization of AChE was diffuse in cytoplasma, in contrast to neuronal cell, where plasma membrane- and neurite-associated AChE expression was revealed (16).

Cholinergic markers appear even in developing tissues during early presynaptic stages. In murine embryonic stem cell line the expression of ChAT, AChE and BChE was observed. Moreover, inhibition of BChE activity by a specific inhibitor led to the decreased proliferation of these cells. Due to these findings, it was concluded, that ACh might act as a regulator of cell proliferation (14). The same function of ACh was possibly observed in human colon cancer cell line HT-29 where the expression of ChAT, AChE and of the $\alpha 7$ subunit of the nicotinic acetylcholine receptor (nAChR) was proved and where nicotine caused an increase of the cell proliferation meanwhile a selective $\alpha 7$ nicotinic acetylcholine receptor antagonist, methyllycaconitine (MLA) reversed the effect of nicotine (17). Choi et al. suggested on the basis of their experimental work on a cholinergic synapse-forming cell line NG108-15 that AChE could play a role in the formation of neuromuscular junctions (18).

\section{AChE and Alzheimer disease}

One of the most consistent neurotransmitter alterations found in brain of AD patients is a loss of the cholinergic markers (19). Some studies have shown that there are changes in the relative proportions of different isoforms of $\mathrm{AChE}$ in the brains of $\mathrm{AD}$ patients and that tetrameric globular G4 isoform is selectively lost from the number of cortical and subcortical brain regions in the postmortem examined AD patients $(2,20)$. This isoform represents approximately $80 \%$ of the total brain AChE (2). The selective loss of G4 isoform leads to the decreased ratio G4/G1. Several studies aimed on the significance of activity and molecular forms changes in $\mathrm{AD}$ shows that $\mathrm{AChE}$ is differently glycosylated in $\mathrm{AD}$ 
patients compared with AChE from non-AD groups, including patients with other forms of dementia (21). A correlation was found between the expression of the amyloid $\beta(A \beta)$, the levels of AChE and abnormally glycosylated AChE in the AD suffered patients (19). In transgenic mice overexpressing $A \beta$, a significant increase of globular monomeric amphiphilic AChE form was found $(19,22)$. Experiments on cell cultures showed that $A \beta$ has enhanced the expression of $\mathrm{AChE}$, thus interfering with calcium homeostasis. This suggests that increased G1 AChE levels around amyloid plaques may be a direct consequence of disturbances in calcium homeostasis caused by $A \beta$ deposition (22).

The cortical AChE activity presented in AD brain was found to be associated with neuritic plaques, in which it is co-localized with $\mathrm{A} \beta$ deposits. It includes both pre-amyloid diffuse deposits and the senile plaques. The incubation of mixture containing $\mathrm{A} \beta$ peptide and $\mathrm{AChE}$ leads to an aggregation in a greater extent (three-fold more) than the $A \beta$ alone (23). Similar results were obtained independently using the different molecular forms of AChE G1, G2 and G4. In all different conditions, $\mathrm{AChE}$ increased the ratio of $\mathrm{A} \beta$ deposition. On the other hand, in $\mathrm{APP}_{\mathrm{SWE}}$ /PSEN1dE9 mice developing $A \beta$ plaques, there was not noticed any $\mathrm{AChE}$ activity in the plaques however BChE activity was present (24).

$\mathrm{A} \beta$ aggregation accelerated by $\mathrm{AChE}$ was observed in presence of an active site inhibitor edrophonium, but it was slowed down in presence of propidium, a PAS binding ligand. For this reason, the induction of aggregation is presumed to be caused by the interaction with AChE PAS (23). In another experimental work, propidium, decamethonium and donepezil, compounds acting as mixed or non-competitive $\mathrm{AChE}$ inhibitors, were proved to inhibit $\mathrm{A} \beta$ aggregation induced by recombinant human $\mathrm{AChE}$ in contrast to edrophonium (25).

Another typical feature of AD, hyperphosprorylated tau (P-tau) protein, is closely related to AChE as well. In VLW human tau transgenic mice, there was observed increased AChE activity, level of AChE protein expression and level of AChE mRNA expression in comparison with wild type mice. The activity and expression of choline acetyltransferase, the enzyme responsible for ACh synthesis, were not altered in the transgenic mice (26).

\section{How can AChE be affected by recent anti-AD therapy?}

The mostly used therapy of AD is based on enhancing cholinergic function using AChE inhibitors. Tacrine was the first drug approved for the treatment of $\mathrm{AD}$ (27). It is a centrally acting $\mathrm{AChE}$ and $\mathrm{BChE}$ inhibitor. In $\mathrm{AD}$ patients receiving tacrine inhibition of $\mathrm{AChE}$, increased release and synthesis of $\mathrm{ACh}$, increased levels of monoamine metabolites, increased numbers of nicotine brain receptors, stimulated glucose metabolism and improved neuropsychological performance was reported (28). Tacrine directed to the active site of cholinesterases prevents substrate binding by occupying access to the site (29). In present tacrine is withdrawn from the market in most countries. The dose-dependent hepatoxicity along with gastrointestinal side effects was the major reason for the withdrawn (30).

Except memantine, which acts as $N$-methyl-d-aspartate receptors antagonist (31), three AChE inhibitors - donepezil, galantamine and rivastigmine - are commercially available for AD therapy. Donepezil is selective reversible AChE inhibitor which does not interact directly with either the catalytic triad or the oxyanion hole but which is orientated along the active-site gorge, extending from the anionic subsite of the active site to the peripheral anionic site via aromatic stacking interactions with conserved aromatic acid residues (32).

Galantamine hydrobromide is a tertiary alkaloid which has a dual mode of action. It is a competitive inhibitor of $\mathrm{AChE}$ and simultaneously it is a positive allosteric modulator of nAChRs (33).

In contrast to other AChE inhibitors used in therapy of $\mathrm{AD}$, carbamylating agent rivastigmine is classified as an intermediate-acting or pseudo-irreversible agent due to its long inhibition on AChE lasting up to 10 hours $(34,35)$. Rivastigmine is an inhibitor of the both $\mathrm{AChE}$ and $\mathrm{BChE}$, meanwhile donepezil and galantamine are selective $\mathrm{AChE}$ inhibitors (36).

To observe $\mathrm{AChE}$ alterations in $\mathrm{AD}$ patients, $\mathrm{AChE}$ present in cerebrospinal fluid (CSF) is often used for experimental purposes, because for several it is believed that $\mathrm{AChE}$ in the CSF originates from neurons of central nervous system (35). Increases level of AChE in the CSF have been reported after treatment with tacrine, galantamine, and donepezil $(35,37-40)$. Not only increase of AChE activity in CSF, but also decrease of G1 and G2 forms and increase of amyloid $\beta_{1-42}$ in CSF of AD patients after treatment with donepezil was described by García-Ayllón et al. (41). Darreh-Shori et al. observed clearly different expression of two AChE splice variants in AD patients treated with tacrine and rivastigmine. Levels of AChE variants differed also in comparison to untreated AD patients (39).

Davidsson et al. observed mild non-significant decline of AChE activity in a cerebrospinal fluid (CSF) of AD patients compared to a healthy controls. After treatment with donepezil and galantamine, there was found a significant increase in $\mathrm{AChE}$ activity. In contrast to galantamine, the rise of AChE activity caused by donepezil was significantly higher and it was in a dose-dependent manner. The difference could be explained by dissimilar mechanism of action (37). More noticeable difference in AChE activity was found between donepezil and rivastigmine after a long-lasting treatment. Meanwhile donepezil caused the increase of AChE activity in CSF, as was described in the work mentioned previously, treatment with rivastigmine led to a significant decline of the activity in comparison with the status before the treatment started. No changes in BChE activity were noticed in both donepezil and rivastigmine treated groups of patients. 
Another interesting fact is that both treatments induced a relative increase of $\mathrm{G} 4 \mathrm{AChE}$ isoform as opposite to a reduction of G1-G2 isoforms. This change means a partial renewal of physiologic conditions altered by $\mathrm{AD}$ (42).

In mammalian brain, there are presented some types of feedback regulation of AChE production. Organophosphorous insecticide chlorpyrifos administered to laboratory rats caused a significant dose-dependent fall of the brain AChE activity, but the level of AChE mRNA remained unchanged and AChE protein level was even higher. The possible explanation of this phenomenon is an inhibitor induced increase in AChE synthesis or a reduced rate of AChE degradation due to inhibition of protease activity in the brain (43). After a prolonged treatment with $\mathrm{AD}$ drug donepezil, the rise of AChE activity and also of AChE mRNA level was proved in brains of laboratory animals. This finding suggests AChE up-regulation induced by prolonged treatment with AChE inhibitors (44). Kaufer et al. noticed that inhibitors of AChE could regulate acetylcholine availability due to bidirectional modulation of genes (45).

On the other hand, rivastigmine is responsible for decline of AChE activity in CSF of AD patients $(35,38,42,46)$. As described in previous section, rivastigmine differs a lot from other inhibitors used in AD therapy by its structure and a mode of inhibition as well. It works due to carbamylating the active site of both $\mathrm{AChE}$ and $\mathrm{BChE} \mathrm{(35).} \mathrm{In} \mathrm{Sweden,} \mathrm{another}$ carbamylating agent, phenserine was studied as a possible drug for $\mathrm{AD}$ treatment. Phenserine treatment was associated with an improvement in cognition and an increase in regional cerebral metabolic rate for glucose (47). Phenserine treatment was also shown to be able to reverse donepezilinduced elevation of AChE expression (35).

As was mentioned above, peripheral anionic site can interact with $\mathrm{A} \beta$ facilitating $\mathrm{A} \beta$ plaques creation. The effect of cholinesterase inhibitors such as donepezil on the formation of amyloid deposition is therefore interesting. The interest of donepezil is in the fact that it does not interact with the active center of the enzyme, but with the peripheral anionic site (19). The elevated AChE protein expression caused by the long-term donepezil treatment may act against its ability to reduce $A \beta$ aggregation. Based on these findings Dareh-shori et al. suggested additional treatment with low doses of the carbamylating agent like rivastigmine, which produces stable AChE inhibition with no significant alteration in $\mathrm{AChE}$ protein expression, in patients taking long-term donepezil treatment (35).

Moreover, AChE inhibitors can probably affect other disease-related mechanisms. Tacrine, donepezil and galantamine, similarly like nAChR agonists nicotine and epibatidine were found to be able to increase level of tau protein in human neuroblastoma SH-SY5Y cells. For the reason that AChE inhibitors worked in a similar way as nAChR agonists and the effect was prevented by the antagonists $d$-tubocurarine and mecamylamine and not prevented by atropine, the results indicate interaction with $\mathrm{nAChR}$ rather than AChE inhibition (48).

\section{Possibilities of AChE expression in cells measurement}

Because AChE levels are routinely estimated enzymatically using various modifications of the Ellman et al. method (49), and are not normally estimated immunochemically and histologically, little information is available on this inactive pool of AChE in pathological and non-pathological conditions (50).

Enzymatic assays are mostly used for AChE determination, nevertheless immunochemical methods for a quantitative protein assessment are available for a long time $(39,51)$. The determination of AChE mRNA level is another possibility how to monitor AChE expression (44). In our opinion, observation of the level of AChE protein expression in patients treated with $\mathrm{AChE}$ inhibitors would be useful tool for the elucidation of their effects in the organism and for prediction of further development of the disease.

The cholinergic nervous system is a complex framework where many processes occur at the same time and influence each other. In such a complicated surrounding it is always difficult to decide what the causation is and what the consequence. For this reason the introduction of some suitable simple model can be useful. Neuroblastoma-derived T 28 hybrid cells are an example of cell line producing AChE. They were used by Lazar et al. for observation of AChE metabolism. The method of de novo synthesized AChE determination was based on cultivation in medium containing heavy isotope labeled amino acids (52). AChE is expressed in various cell types over the all organism and similarly various cell lines expressing AChE are commercially available, for example murine neuroblastoma cells SN-56, N1E-115, NB41A3 or Neuro-2a $(53,54)$. The cholinergic murine neuroblastoma cells $\mathrm{SN}-56$ derived from septal neurons were used as a model of cholinergic neurons from basal forebrain to evaluate the cholinesterase dependent mechanism of cadmium neurotoxicity (53). The observation of AChE inhibitors influence on the activity and especially protein levels of AChE in AChE producing cells could provide valuable knowledge leading to increase of the efficiency in the therapy with AChE inhibitors.

\section{Conclusion}

In conclusion we can say, that AChE is involved in various processes in organism, that include, except the termination of cholinergic transmission, also apoptosis, cell cycle regulation and processes related to the AD pathogenesis. For this reason, AChE inhibitors used in therapy of $\mathrm{AD}$ can have more complex effect. Opinion, that treatment with AChE inhibitors is not only symptomatic but also disease-modifying, becomes more and more widespread. There are numerous evidences showing that inhibitors of AChE influence the level of AChE expression and protein mass except the activity. However, very little is known about effect of AChE inhibitors on AChE production on cell level. 
Studies aimed on this topic can elucidate a lot about the course of AD therapy.

\section{Acknowledgements}

The Ministry of Education, Youth and Sports of the Czech Republic is gratefully acknowledged for project SV/ FVZ201203.

\section{References}

1. Shen ZX. Brain cholinesterases: II. The molecular and cellular basis of Alzheimer's disease. Med Hypotheses 2004; 63: 308-21.

2. Small DH, Michaelson S, Sberna G. Non-classical actions of cholinesterases: Role in cellular differentiation, tumorigenesis and Alzheimer's disease. Neurochem Int 1996; 28 : 453-83.

3. Medeiros R, Baglietto-Vargas D, LaFerla FM. The role of Tau in Alzheimer's disease and related disorders CNS. Neurosci Ther 2011; 17: 514-24.

4. Weiner MW, Aisen PS, Jack CR et al. The Alzheimer's disease neuroimaging initiative: progress report and future plans. Alzheimers Dement 2010; 6: 202-11.

5. Mao P, Reddy PH. Aging and amyloid beta-induced oxidative DNA damage and mitochondrial dysfunction in Alzheimer's disease: Implications for early intervention and therapeutics. BBA Mol Basis Dis 2011; 1812: 1359-70.

6. Dvir H, Silman I, Harel M, Rosenberry TL, Sussman JL. Acetylcholinesterase: From 3D structure to function. Chem Biol Interact 2010; 187: 10-22.

7. Darvesh S, Hopkins DA, Geula C. Neurobiology of butyrylcholinesterase. Nat Rev Neurosci 2003; 4: 131-8.

8. Dougherty DA, Stauffer DA. Acetylcholine binding by a synthetic receptor: implications for biological recognition. Science 1990; 250: 1558-60.

9. Kozurkova M, Hamulakova S, Gazova Z, Paulikova H, Kristian P. Neuroactive multifunctional tacrine congeners with cholinesterase, anti-amyloid aggregation and neuroprotective properties. Pharmaceuticals 2011; 4: 382-418.

10. Craig LA, Hong NS, McDonald RJ. Revisiting the cholinergic hypothesis in the development of Alzheimer's disease. Neurosci Biobehav R 2011; 35: 1397-409.

11. Pang YP, Quiram P, Jelacic T, Hong F, Brimijoin S. Highly potent, selective, and low cost bis-tetrahydroaminacrine inhibitors of acetylcholinesterase. Steps toward novel drugs for treating Alzheimer's disease. J Biol Chem 1996; 271: 23646-9.

12. Francis PT, Palmer AM, Snape M, Wilcock GK. The cholinergic hypothesis of Alzheimer's disease: a review of progress. J Neurol Neurosur Ps 1999; 66: 137-47.

13. Chatonnet A, Lockridge O. Comparison of butyrylcholinesterase and acetylcholinesterase. Biochem J 1989; 260: 625-34.

14. Paraoanu LE, Steinert G, Koehler A, Wessler I, Layer PG. Expression and possible functions of the cholinergic system in a murine embryonic stem cell line. Life Sci 2007; 80: 2375-9.

15. Zhang XJ, Yang L, Zhao Q et al. Induction of acetylcholinesterase expression during apoptosis in various cell types. Cell Death Differ 2002; 9: 790-800.

16. Thullbery MD, Cox HD, Schule T, Thompson CM, George KM. Differential localization of acetylcholinesterase in neuronal and non-neuronal cells. J Cell Biochem 2005; 96(3): 599-610.

17. Pettersson A, Nilsson L, Nylund G, Khorram-Manesh A, Nordgren S, Delbro DS Is acetylcholine an autocrine/paracrine growth factor via the nicotinic $\alpha$ 7-receptor subtype in the human colon cancer cell line HT-29? Eur J Pharmacol 2009; 609: 27-33.

18. Choi RCY, Yam SCY, Hui B, Wan DCC, Tsim KWK. Over-expression of acetylcholinesterase stimulates the expression of agrin in NG108-15 cells. Neurosci Lett 1998; 248: 17-20.

19. Talesa VN. Acetylcholinesterase in Alzheimer's disease. Mech Ageing Dev 2001; 122: 1961-9.

20. Shah RS, Lee HG, Xiongwei Z, Perry G, Smith MA, Castellani RJ. Current approaches in the treatment of Alzheimer's disease. Biomed Pharmacoter 2008; 62: 199-207.

21. Bonham JR, Dale G, Scott DJ, Wagget J, Atack JR. The characterization of molecular forms of acetylcholinesterase in Hirschprung's disease. Clin Chim Acta 1999; 171: $263-9$.

22. Fodero LR, Sáez-Valero J, McLean CA et al. Altered glycosylation of acetylcholinesterase in APP (SW) Tg2576 transgenic mice occurs prior to amyloid plaque deposition. J Neurochem 2002; 81: 441-8.

23. Inestrosa NC, Alvarez A, Pérez CA et al. Acetylcholinesterase accelerates assembly of amyloid- $\beta$-peptides into Alzheimer's fibrils: Possible role of the peripheral site of the enzyme. Neuron 1996; 16: 881-91.

24. Darvesh S, Cash MK, Andrew Reid G, Martin E, Mitnitski A, Geula C. Butyrylcholinesterase is associated with $\beta$-Amyloid plaques in the transgenic $\mathrm{APP}_{\mathrm{SWE}}$ PSEN1dE9 mouse model of Alzheimer disease. J Neuropathol Exp Neurol 2012; 71(1): $2-14$
25. Bartolini M, Bertucci C, Cavrini V, Adrisano V. $\beta$-Amyloid aggregation induced by human acetylcholinesterase: inhibition studies. Biochem Pharmacol 2003; 65: $407-16$.

26. Silveyra MX, García-Ayllón MS, de Barreda EG et al. Altered expression of brain acetylcholinesterase in FTDP-17 human tau transgenic mice. Neurobiol Aging 2012; 33: 624.e23-34

27. Crismon ML. Tacrine: first drug approved for Alzheimer's disease. Ann Pharmacother 1994; 28(6): 744-51.

28. Wagstaff AJ, McTavish D. Tacrine. Drug \& Aging 1994; 4(6): 510-40.

29. Alonso D, Dorronsoro I, Rubio L et al. Donepezil-tacrine hybrid related derivatives as new dual binding site inhibitors of AChE. Bioorg Med Chem 2005; 13 : 6588-97.

30. Luppi B, Bigucci F, Corace G et al. Albumin nanoparticles carrying cyclodextrins for nasal delivery of the anti-Alzheimer drug tacrine. Eur J Pharm Sci 2011; 44(4) 559-65.

31. Johnson JW, Kotermanski SE. Mechanism of action of memantine. Curr Opin Pharmacol 2006; 6(1): 61-7.

32. Kryger G, Silman I, Sussman JL. Structure of acetylcholinesterase complexed with E2020 (Aricept): implications for the design of new anti-Alzheimer drugs. Structure 1999; 7(3): 297-307.

33. Lilienfeld S. Galantamine - a novel cholinergic drug with a unique dual mode of action for the treatment of patients with Alzheimer's disease. CNS Drug Rev 2002; 8(2): 159-76.

34. Jann MW. Rivastigmine, a new-generation cholinesterase inhibitor for the treatment of Alzheimer's disease. Pharmacotherapy 2000; 20(1): 1-12.

35. Darreh-Shori T, Hosseini SM, Nordberg A. Pharmacodynamics of cholinesterase inhibitors suggests add-on therapy with a low-dose carbamylating inhibitor in patients on long-term treatment with rapidly reversible inhibitors. J Alzheimers Dis 2014; 39: 423-40.

36. Bullock R. The clinical benefits of rivastigmine may reflect its dual inhibitory mode of action: an hypothesis. Int J Clin Pract 2002; 56(3): 206-14.

37. Davidsson P, Blennow K, Andreasen N, Eriksson B, Minthon L, Hesse C. Differential increase in cerebrospinal fluid-acetylcholinesterase after treatment with acetylcholinesterase inhibitors in patients with Alzheimer's disease. Neurosci Lett 2001; 300: 157-60

38. Parnetti L, Amici S, Lanari A et al. Cerebrospinal fluid levels of biomarkers and activity of acetylcholinesterase (AChE) and butyrylcholinesterase in $\mathrm{AD}$ patient before and after treatment with different AChE inhibitors. Neurol Sci 2002; 23: 95-96.

39. Darreh-Shori T, Hellström-Lindahl E, Flores-Flores C, Guan ZZ, Soreq H, Nordberg A. Long-lasting acetylcholinesterase splice variations in anticholinesterase-treated Alzheimer's disease patients. J Neurochem 2004; 88: 1102-13.

40. Darreh-Shori T, Meurling L, Pettersson T. et al. Changes in the activity and protein levels of CSF acetylcholinesterases in relation to cognitive function of patients with mild Alzheimer's disease following chronic donepezil treatment. J Neural Transm 2006; 113: 1791-801.

41. García-Ayllón MS, Silveyra MX, Andreasen N, Brimijoin S, Blennow K, Sáez-Valero J. Cerebrospinal fluid acetylcholinesterase changes after treatment with donepezil in patients with Alzheimer's disease. J Neurochem 2007; 101: 1701-11.

42. Amici S, Lanari A, Romani R, Antognelli C, Gallai V, Parnetti L. Cerebrospinal fluid acetylcholinesterase activity after long-term treatment with donepezil and rivastigmina. Mech Ageing Develop 2001; 122: 2057-62.

43. Chiappa S, Padilla S, Koenigsberger C, Moser V, Brimijoin S. Slow accumulation of acetylcholinesterase in rat brain during enzyme inhibition by repeated dosing with chlorpyrofos. Biochem Pharmacol 1995; 19(7): 955-63.

44. Zivin M, Pregelj P. Prolonged treatment with donepezil increases acetylcholinesterase expression in the central nervous system. Psychiatr Danub 2008; 20(2): $168-73$

45. Kaufer D, Friedman A, Seidman S, Soreq H. Acute stress facilitates long-lasting changes in cholinergic gene expression. Nature 1998; 393:373-7.

46. Cutler NR, Polinsky RJ, Srarnek JJ et al. Dose-dependent CSF acetylcholinesterase inhibition by SDZ ENA 713 in Alzheimer's disease. Acta Neurol Scand 1998; 97(4): 244-50.

47. Kadir A, Andreasen N, Almkvist O et al. Effect of phenserine treatment on brain functional activity and amyloid in Alzheimer's disease. Ann Neurol 2008; 63: 621-631.

48. Hellström-Lindahl E, Moore H, Nordberg A. Increased levels of Tau protein in SHSY5Y cells after treatment with cholinesterase inhibitors and nicotinic agonists. J Neurochem 2000; 74(2): 777-84.

49. Ellman GL, Courtney KD, Andres V, Feather-Stone RM. A new and rapid colorimetric determination of acetylcholinesterase activity. Biochem Pharmacol 1961; 7: $88-95$.

50. García-Ayllón MS, Small DH, Avila J, Sáez-Valero J. Revisiting the role of acetylcholinesterase in Alzheimer's disease: cross-talk with P-tau and $\beta$-amyloid. Front Mol Neurosci 2011; 4: 22

51. Nørgaard-Pedersen B, Hangaard J, Bjerrum OJ. Quantitative enzyme antigen immunoassay of acetylcholinesterase in amniotic fluid. Clin Chem 1983;29(6): 1061-4. 
52. Lazar M, Salmeron E, Vigny M, Massoulié J. Heavy isotope-labeling study of the metabolism of monomeric and tetrameric acetylcholinesterase forms in the murine neuronal-like T 28 hybrid cell line. J Biol Chem 1984; 259: 3703-13.

53. Del Pino J, Zeballos G, Anadon MJ et al. Higher sensitivity to cadmium induced cell death of basal forebrain cholinergic neurons: A cholinesterase dependent mechanism. Toxicology 2014; 325: 151-9.

54. ATCC. Products. Cells and microorganisms. (accessed March 12, 2015 on http:// www.lgcstandards-atcc.org/Products/Cells_and_Microorganisms.aspx)

Received: $17 / 03 / 2015$

Accepted in revised form: 27/05/2015

\section{Corresponding author:}

Assoc. Prof. Miroslav Pohanka, RNDr., Ph.D., Dsc., Department of Molecular Pathology and Biology, Faculty of Military Health Sciences, University of Defense, Třebešská 1575, 500 01, Hradec Králové, Czech Republic; e-mail: miroslav .pohanka@unob.cz 